\title{
Traveling Curved Fronts of Anisotropic Curvature Flows
}

\author{
Yoshiko Marutani*, Hirokazu NinOmiYA* \\ and Rémi WEIDENFELD ${ }^{\dagger}$ \\ * Department of Applied Mathematics and Informatics, \\ Ryukoku University, Seta, Otsu 520-2194, Japan \\ † Ecole Centrale de Lyon, Dept. MI, CNRS UMR 5585, \\ 36, Avenue Guy de Collongues 69134 Ecully, France
}

Received May 6, 2005

Revised October 19, 2005

\begin{abstract}
In this paper, the anisotropic curvature flows with driving force are considered. The existence of traveling curved fronts is shown by constructing supersolutions and subsolutions. By the advantage of this method, their global stability is also proved. In the last section the profiles of the traveling fronts are discussed when the anisotropy becomes strong and converges to a non-smooth function.
\end{abstract}

Key words: traveling wave, curvature flow, anisotropy

\section{Introduction}

The dynamics of the phase boundaries is one of the interesting problems in applied mathematics. The interface between the two physical states is mainly controlled by the surface free energy and the energy difference between two bulk phases. The surface free energy usually depends on the orientation: it is represented by a function $\Psi(\theta)$ with period $\pi$ and where $\theta$ is the angle between the $x$ axis and the normal vector (cf. $[11,14])$. Let denote by $\Gamma_{t}$ the interface at time $t, V_{n}$ the normal velocity of the interface and $\kappa$ its curvature. In this paper we consider the following moving boundary problem in two-dimensional space $(N=2)$ :

$$
\left\{\begin{array}{l}
V_{n}=-\Psi(\theta)\left(\Psi(\theta)+\Psi^{\prime \prime}(\theta)\right) \kappa+a \Psi(\theta) \\
\left.\Gamma_{t}\right|_{t=0}=\Gamma_{0},
\end{array}\right.
$$

where $a$ is a constant which corresponds to the energy difference between the two states. This equation was considered by Angenent and Gurtin [1] (also see [3, 4, 11] for instance). The anisotropic curvature flow (1.1) fits to a Finsler metric, see Section 2 for more details.

Through this work, we assume that

(H1) $\Psi \in C^{2}(\mathbb{R})$ is $\pi$-periodic and $\Psi^{\prime \prime}$ is a globally Lipschitz function,

(H2) there exist positive constants $\lambda_{i}(i=1,2,3,4)$ such that for all $\theta \in \mathbb{R}$

$$
\lambda_{1} \leq \Psi(\theta) \leq \lambda_{2}, \quad \lambda_{3} \leq \Psi(\theta)+\Psi^{\prime \prime}(\theta) \leq \lambda_{4} .
$$


If the interface $\Gamma_{t}$ is represented by the level set of $U$, that is,

$$
\Gamma_{t}=\{(x, y) \mid U(x, y, t)=0\},
$$

then $U$ satisfies the following degenerate parabolic equation:

$$
U_{t}=-\Phi^{0}(\nabla U)\left\{-\sum_{i, j} \frac{\partial^{2} \Phi^{0}}{\partial p_{i} \partial p_{j}}(\nabla U) \frac{\partial^{2} U}{\partial x_{i} \partial x_{j}}+a\right\}
$$

where $\Phi^{0}$ is a function related to $\Psi$ (see Section 2). Moreover, if $\Gamma_{t}$ is a graph, then we may set $U(x, y, t)=y-u(x, t)$. Denoting the angle between the normal vector $\left(-u_{x}, 1\right)$ and the $x$ axis by $\theta\left(u_{x}\right)$ and setting

$$
G_{1}\left(u_{x}\right):=\Psi\left(\theta\left(u_{x}\right)\right)\left(\Psi\left(\theta\left(u_{x}\right)\right)+\Psi^{\prime \prime}\left(\theta\left(u_{x}\right)\right)\right), \quad G_{2}\left(u_{x}\right):=a \Psi\left(\theta\left(u_{x}\right)\right) \sqrt{1+u_{x}^{2}},
$$

we see that $u$ satisfies the following parabolic equation:

$$
\begin{cases}u_{t}=\frac{G_{1}\left(u_{x}\right)}{1+u_{x}^{2}} u_{x x}+G_{2}\left(u_{x}\right) & \text { in } \mathbb{R} \times(0, \infty) \\ u(x, 0)=u_{0}(x) & \text { in } \mathbb{R} .\end{cases}
$$

In this work, we are mainly interested in traveling curved fronts of (1.3) when $a>0$ and the initial function enjoys linear growth conditions at $x \rightarrow \pm \infty$. We prove that for any pair of two asymptotic lines at infinity there exists a traveling curved front solution of problem (1.3), which is a solution of the form $\varphi\left(x-c_{1} t\right)+c_{2} t$ with suitable constants $c_{1}$ and $c_{2}$. Then we prove that this solution is stable in the following sense: if $u$ is any solution possessing two asymptotic lines, it converges for large time to the traveling curved front which has the same asymptotic lines as $u$. The existence of the traveling curved fronts and the global stability for the isotropic case $\Psi \equiv 1$ are studied in $[8,12]$.

The organization of this paper is as follows: in Section 2, we recall the definition of a Finsler metric. Then, in Section 3, we derive an existence result for problem (1.3) as well as a comparison principle. The main section of this paper is Section 4 where we prove the existence of a traveling curved front solution and study its stability. In Sections 5 and 6, we give two applications of our main result: first we prove a stability property of the traveling curved front in the class of non graph solutions; then we prove existence of traveling curved front solution in the case of non-smooth convex anisotropy.

\section{A Short Overview on Finsler Geometry}

We first recall the definition of a Finsler metric.

Definition. A continuous function $\Phi: \mathbb{R}^{N} \rightarrow[0, \infty)$ is a Finsler metric if (i) $\Phi \in C^{2}\left(\mathbb{R}^{N} \backslash\{0\}\right)$ is convex and $\nabla^{2} \Phi$ is locally Lipschitz; 
(ii) $\Phi^{2}$ is strictly convex;

(iii) $\Phi$ is even and positively homogeneous of degree one:

$$
\Phi(s \xi)=|s| \Phi(\xi) \quad \text { for all } s \in \mathbb{R} \text { and } \xi \in \mathbb{R}^{N} ;
$$

(iv) $\Phi$ is a bounded and coercive map:

$$
\lambda|\xi| \leq \Phi(\xi) \leq \Lambda|\xi|
$$

for all $\xi \in \mathbb{R}^{N}$ and for two positive constants $\lambda$ and $\Lambda$.

We recall that the dual function defined by $\Phi^{0}(\xi)=\sup \left\{\xi^{*} \cdot \xi \mid \Phi\left(\xi^{*}\right) \leq 1\right\}$ is also a Finsler metric and it holds that for all $\xi \in \mathbb{R}^{N}$

$$
\begin{aligned}
& \Phi^{0}(s \xi)=|s| \Phi^{0}(\xi), \\
& \lambda^{0}|\xi| \leq \Phi^{0}(\xi) \leq \Lambda^{0}|\xi|,
\end{aligned}
$$

with $\lambda^{0}=1 / \Lambda$ and $\Lambda^{0}=1 / \lambda$.

In the particular case of dimension $N=2,(2.3)$ implies that for all $\xi \neq 0$ we have

$$
\Phi^{0}(\xi)=|\xi| \Phi^{0}\left(\frac{\xi}{|\xi|}\right),
$$

and that there exists a $\pi$-periodic function $\Psi$ satisfying

$$
\Phi^{0}(\xi)=|\xi| \Psi(\theta)
$$

where $\theta$ is the angle between $\xi$ and the $x$ axis. Moreover, the regularity properties on $\Phi^{0}$ directly implies (H1) and (2.4) gives the first inequality in (H2). One proves the second inequality in (H2), using

$$
\Phi_{\xi \xi}^{0}(\xi)=\frac{\Psi(\theta)+\Psi^{\prime \prime}(\theta)}{|\xi|}\left(\begin{array}{cc}
\sin ^{2} \theta & -\sin \theta \cos \theta \\
-\sin \theta \cos \theta & \cos ^{2} \theta
\end{array}\right) .
$$

See [4] for more details.

Next we derive the parabolic equation for motion by anisotropic mean curvature. If $\Gamma$ is a smooth hypersurface of $\mathbb{R}^{N}$ and $n$ its outer normal vector (in the Euclidean sense), the $\Phi$-normal vector and the $\Phi$-mean curvature are defined respectively by

$$
n_{\Phi}=\Phi_{\xi}^{0}(n), \quad \kappa_{\Phi}=\frac{1}{N-1} \operatorname{div} n_{\Phi} .
$$

The level set formulation is the following: if $U$ is a smooth function with non vanishing gradient such that $\Gamma=\left\{x \in \mathbb{R}^{N} \mid U\left(x_{1}, \ldots, x_{N}\right)=0\right\}$, and $U$ changes of sign along $\Gamma$, then

$$
\begin{aligned}
& n=\frac{\nabla U}{|\nabla U|}, \quad n_{\Phi}=\Phi_{\xi}^{0}(\nabla U), \\
& \kappa=\frac{1}{N-1} \operatorname{div} \frac{\nabla U}{|\nabla U|}, \quad \kappa_{\Phi}=\frac{1}{N-1} \operatorname{div} \Phi_{\xi}^{0}(\nabla U),
\end{aligned}
$$


on $\Gamma$. If $N=2,(2.6)$ implies that

$$
\kappa_{\Phi}=\left(\Psi(\theta)+\Psi^{\prime \prime}(\theta)\right) \kappa
$$

We also define the normal velocity and the anisotropic normal velocity by

$$
V_{n}=-\frac{U_{t}}{|\nabla U|}, \quad V_{n, \Phi}=-\frac{U_{t}}{\Phi^{0}(\nabla U)},
$$

respectively. Then we can get the natural moving boundary problem

$$
V_{n, \Phi}=-(N-1) \kappa_{\Phi}+a,
$$

especially, in dimension $N=2$,

$$
V_{n, \Phi}=-\kappa_{\Phi}+a
$$

By (2.5) and (2.7), (2.9) is transformed into

$$
V_{n}=-\Psi(\theta)\left(\Psi(\theta)+\Psi^{\prime \prime}(\theta)\right) \kappa+a \Psi(\theta) .
$$

Using the level set formulation, we can also obtain (1.2).

\section{Existence and Comparison Principle}

In this section, we use a result of Barles, Biton, Bourgoing and Ley [2] to prove the existence as well as a comparison principle for Problem (1.3) when the initial function $u_{0}$ has a limited growth at infinity (see condition (H3) below). To that purpose, we use viscosity solutions of $(1.3)$. We refer to $[7, \S 8]$ for a definition of such solutions. Note that a classical solution is a viscosity solution (see $[7, \S 1]$ for instance). The same remark holds for supersolutions and subsolutions.

First, let define the continuous function $\sigma$ by

$$
\sigma(p)=\sqrt{\frac{G_{1}(p)}{1+p^{2}}},
$$

and we check that the hypotheses of [2] are fulfilled. Namely, we prove that there exist two positive constants $C_{1}, C_{2}$ such that for all $p, q \in \mathbb{R}$

$$
\begin{aligned}
|\sigma(p)-\sigma(q)| & \leq \frac{C_{1}|p-q|}{1+|p|+|q|}, \\
\left|G_{2}(p)-G_{2}(q)\right| & \leq C_{2}|p-q| .
\end{aligned}
$$

Note that

$$
\theta(p)=\operatorname{Arccos}\left(\frac{-p}{\sqrt{1+p^{2}}}\right)
$$


is a globally Lipschitz continuous function. A simple computation shows that $\theta^{\prime}(p)=1 /\left(1+p^{2}\right)$. Then, by (H1), it holds that the function $p \mapsto G_{1}(p)$ is globally Lipschitz. Note also that $p \mapsto G_{2}(p)$ is globally Lipschitz so that (3.2) is satisfies.

Proof of (3.1). First, by (H2), there exists $c$ such that for all $p \in \mathbb{R}$

$$
\lambda_{1} \lambda_{3} \leq G_{1}(p) \leq \lambda_{2} \lambda_{4}
$$

Then for all $p, q \in \mathbb{R}$, it holds that

$$
\left|\sqrt{G_{1}(p)}-\sqrt{G_{1}(q)}\right|=\frac{\left|G_{1}(p)-G_{1}(q)\right|}{\sqrt{G_{1}(p)}+\sqrt{G_{1}(q)}} \leq \frac{1}{2 \sqrt{\lambda_{1} \lambda_{3}}}\left|G_{1}(p)-G_{1}(q)\right|,
$$

and we deduce from the fact that $G_{1}$ is globally Lipschitz that

$$
\left|\sqrt{G_{1}(p)}-\sqrt{G_{1}(q)}\right| \leq C_{3}|p-q|
$$

with some positive constant $C_{3}$. Also remark that there is a positive constant $C_{4}$ such that

$$
\left|\sqrt{1+p^{2}}-\sqrt{1+q^{2}}\right| \leq C_{4}|p-q| .
$$

Without lost of generality we may suppose that $|p| \geq|q|$. By (3.5), (3.3) and (3.6), we have

$$
\begin{aligned}
|\sigma(p)-\sigma(q)| & =\left|\frac{\sqrt{G_{1}(p)}-\sqrt{G_{1}(q)}}{\sqrt{1+p^{2}}}+\sqrt{G_{1}(q)}\left(\frac{1}{\sqrt{1+p^{2}}}-\frac{1}{\sqrt{1+q^{2}}}\right)\right| \\
& \leq \frac{C_{3}|p-q|}{\sqrt{1+p^{2}}}+\frac{C_{5}|p-q|}{\sqrt{\left(1+p^{2}\right)\left(1+q^{2}\right)}} \\
& \leq \frac{C_{6}|p-q|}{1+|p|+|q|}
\end{aligned}
$$

where the last inequality comes from

$$
(1+|p|+|q|)^{2} \leq(1+2|p|)^{2} \leq 6\left(1+|p|^{2}\right),
$$

and

$$
(1+|p|+|q|)^{2} \leq 3\left(1+p^{2}+q^{2}\right) \leq 3\left(1+p^{2}\right)\left(1+q^{2}\right) .
$$

This proves the equation (3.1).

Now, we give the precise hypothesis on the initial function $u_{0}$ :

(H3) There exist $\nu \in[0,(1+\sqrt{5}) / 2)$ and a modulus of continuity $m$ such that

$$
\left|u_{0}(x)-u_{0}(y)\right| \leq m\left((1+|x|+|y|)^{\nu}|x-y|\right) \quad \text { for all } x, y \in \mathbb{R}
$$

where $\lim _{s \rightarrow+0} m(s)=0$ and $m(s+t) \leq m(s)+m(t)$. 
Finally, we introduce the space $\mathcal{C}_{\text {poly }}$ of functions on $\mathbb{R} \times[0, T]$ which have polynomial growth at infinity. More precisely $v \in \mathcal{C}_{\text {poly }}$ if there exists $\ell>0$ such that

$$
\frac{v(x, t)}{1+|x|^{\ell}} \rightarrow 0 \text { as }|x| \rightarrow+\infty \text { uniformly with respect to } t \in[0, T] .
$$

Then we deduce the following Lemma from [2, Theorem 2.1 and Corollary].

LEMMA 3.1. The following hold.

(i) Comparison principle. If $u_{0}$ satisfies (H3) and if $\bar{u} \in \mathcal{C}_{\text {poly }}$ (resp. $\underline{u} \in$ $\left.\mathcal{C}_{\text {poly }}\right)$ is a supersolution (resp. subsolution) of (1.3) with $\bar{u}(x, 0) \geq u_{0}(x)$ for all $x \in \mathbb{R}\left(\right.$ resp. $\left.\underline{u}(x, 0) \leq u_{0}(x)\right)$, then

$$
\underline{u} \leq \bar{u} \text { in } \mathbb{R} \times[0, T] .
$$

(ii) Existence result. Let $u_{0}$ satisfies $(\mathrm{H} 3)$. In $\mathcal{C}_{\text {poly }}$, there exists a unique continuous viscosity solution of (1.3).

\section{The Traveling Curved Fronts}

Consider the solution of

$$
u_{t}=\frac{G_{1}\left(u_{x}\right)}{1+u_{x}^{2}} u_{x x}+G_{2}\left(u_{x}\right) .
$$

DEFINITION. We say that a solution $u$ of $(4.1)$ is a traveling curved front if it holds that $u(x, t)=\varphi\left(x-c_{1} t\right)+c_{2} t$ for all $(x, t) \in \mathbb{R} \times[0,+\infty)$ where there exist $0<$ $\theta_{-}<\theta_{+}<\pi$ such that the function $\varphi$ has two asymptotic lines $y=\tan \left(\theta_{ \pm}-\pi / 2\right) x$ as $x \rightarrow \pm \infty$.

The function $\varphi$ is called the profile of the traveling curved front and the vector $c:={ }^{t}\left(c_{1}, c_{2}\right)$ is the velocity of the front. The profile $\varphi$ satisfies

$$
c_{2}-c_{1} \varphi^{\prime}(x)=\frac{G_{1}\left(\varphi^{\prime}(x)\right) \varphi^{\prime \prime}(x)}{1+\varphi^{\prime}(x)^{2}}+G_{2}\left(\varphi^{\prime}(x)\right) .
$$

Let $\theta(x)$ be the angle between the $x$-axis and the normal vector to the graph of $\varphi$ at the point $x$. Then we have

$$
\varphi^{\prime}(x)=\tan \left(\theta-\frac{\pi}{2}\right),
$$

and (4.2) reduces to

$$
\begin{aligned}
\theta^{\prime}(x) & =f(\theta), \\
\theta(-\infty) & =\theta_{-}, \\
\theta(\infty) & =\theta_{+},
\end{aligned}
$$


where

$$
f(\theta):=\frac{c_{1} \cos \theta+c_{2} \sin \theta-a \Psi(\theta)}{\Psi(\theta)\left(\Psi(\theta)+\Psi^{\prime \prime}(\theta)\right) \sin \theta} .
$$

First we show the following lemma.

Lemma 4.1. For any $\theta_{ \pm}\left(0<\theta_{-}<\theta_{+}<\pi\right)$, there exists a unique pair of constants $\left(c_{1}, c_{2}\right)$ such that

$$
f\left(\theta_{ \pm}\right)=0 .
$$

Moreover, if $a \neq 0$, then $f(\theta) / a$ does not depend on $a$ and

$$
\begin{cases}\frac{f(\theta)}{a}>0 & \text { for } \theta_{-}<\theta<\theta_{+}, \\ \frac{f(\theta)}{a}<0 & \text { for } 0 \leq \theta<\theta_{-}, \quad \theta_{+}<\theta \leq \pi, \\ \frac{f^{\prime}\left(\theta_{-}\right)}{a}>0, & \frac{f^{\prime}\left(\theta_{+}\right)}{a}<0 .\end{cases}
$$

Proof. By (4.5) and (4.6), $c_{1}$ and $c_{2}$ are uniquely determined as follows:

$$
\begin{aligned}
\left(\begin{array}{l}
c_{1} \\
c_{2}
\end{array}\right) & =a\left(\begin{array}{cc}
\cos \theta_{+} & \sin \theta_{+} \\
\cos \theta_{-} & \sin \theta_{-}
\end{array}\right)^{-1}\left(\begin{array}{c}
\Psi\left(\theta_{+}\right) \\
\Psi\left(\theta_{-}\right)
\end{array}\right) \\
& =-\frac{a}{\sin \left(\theta_{+}-\theta_{-}\right)}\left(\begin{array}{cc}
\sin \theta_{-} & -\sin \theta_{+} \\
-\cos \theta_{-} & \cos \theta_{+}
\end{array}\right)\left(\begin{array}{c}
\Psi\left(\theta_{+}\right) \\
\Psi\left(\theta_{-}\right)
\end{array}\right) .
\end{aligned}
$$

Thus $f(\theta) / a$ does not depend on $a$. Note that $\Psi(\theta)\left(\Psi(\theta)+\Psi^{\prime \prime}(\theta)\right) \sin \theta>0$ for all $\theta \in(0, \pi)$. Then we set

$$
h(\theta):=c_{1} \cos \theta+c_{2} \sin \theta-a \Psi(\theta) .
$$

Since

$$
h^{\prime \prime}(\theta)=-c_{1} \cos \theta-c_{2} \sin \theta-a \Psi^{\prime \prime}(\theta)
$$

we have that

$$
\begin{aligned}
& -h^{\prime \prime}(\theta)-h(\theta)=a\left(\Psi^{\prime \prime}(\theta)+\Psi(\theta)\right), \\
& h\left(\theta_{+}\right)=h\left(\theta_{-}\right)=0 .
\end{aligned}
$$

Let $K(\theta, \xi)$ be the Green function, that is,

$$
K(\theta, \xi):= \begin{cases}\frac{\sin \left(\theta-\theta_{-}\right) \sin \left(\theta_{+}-\xi\right)}{\sin \left(\theta_{+}-\theta_{-}\right)} & \text {for } \theta<\xi, \\ \frac{\sin \left(\xi-\theta_{-}\right) \sin \left(\theta_{+}-\theta\right)}{\sin \left(\theta_{+}-\theta_{-}\right)} & \text {for } \xi<\theta,\end{cases}
$$


so that $h$ is represented as

$$
h(\theta)=a \int_{\theta_{-}}^{\theta_{+}} K(\theta, \xi)\left(\Psi^{\prime \prime}(\xi)+\Psi(\xi)\right) d \xi .
$$

For $\xi \in\left(\theta_{-}, \theta_{+}\right)$, it holds that $K(\theta, \xi)>0$ if $\theta \in\left(\theta_{-}, \theta_{+}\right)$. Since $\Psi^{\prime \prime}(\xi)+\Psi(\xi)>0$, it implies that if $a>0$ (resp. $a<0)$ then $h>0$ (resp. $h<0)$ in $\left(\theta_{-}, \theta_{+}\right)$. Also, we deduce from the Hopf Lemma that $h^{\prime}\left(\theta_{-}\right)>0$ and $h^{\prime}\left(\theta_{+}\right)<0$ if $a>0$ and that $h^{\prime}\left(\theta_{-}\right)<0$ and $h^{\prime}\left(\theta_{+}\right)>0$ if $a<0$. In the case where $\theta_{+}<\theta \leq \pi$ and $a>0$, we have $h^{\prime}\left(\theta_{+}\right)<0, h\left(\theta_{+}\right)=0$ and $h^{\prime \prime}(\theta)+h(\theta)<0$. It follows from the Strum theorem that $h$ possesses no zeros between $\theta_{+}$and $\pi$, which implies that $h<0$ on the interval. We can show the other cases too. The equality

$$
f^{\prime}\left(\theta_{ \pm}\right)=\frac{h^{\prime}\left(\theta_{ \pm}\right)}{\Psi\left(\theta_{ \pm}\right)\left(\Psi\left(\theta_{ \pm}\right)+\Psi^{\prime \prime}\left(\theta_{ \pm}\right)\right) \sin \theta_{ \pm}}
$$

proves the last two inequalities of (4.7).

Angenent and Gurtin [1] already proved the above lemma in the context of the Finsler metric. See [1, Theorem on steady motions, p. 349] or [11, Section 9.2, p. 65].

Lemma 4.2. If $a>0$ and $0<\theta_{-}<\theta_{+}<\pi$, then there exists a unique traveling curved front $u(x, t)=\varphi\left(x-c_{1} t\right)+c_{2} t$ satisfying

$$
\begin{aligned}
& \lim _{x \rightarrow+\infty}\left(\varphi(x)-x \tan \left(\theta_{+}-\pi / 2\right)\right)=0, \\
& \lim _{x \rightarrow-\infty}\left(\varphi(x)-x \tan \left(\theta_{-}-\pi / 2\right)\right)=0 .
\end{aligned}
$$

Moreover, the following holds:

(i) $\varphi^{\prime \prime}(x)>0,0<\varphi(x)-x \varphi^{\prime}(x) \leq \varphi(0)$ for $x \in \mathbb{R}$.

(ii) $\sup _{x \in \mathbb{R}}\left|\varphi^{\prime}(x)\right|<\infty$.

(iii) The vector $c={ }^{t}\left(c_{1}, c_{2}\right)$ belongs to the cone starting at the origin and delimited by the vectors ${ }^{t}\left(\cos \left(\theta_{+}-\pi / 2\right), \sin \left(\theta_{+}-\pi / 2\right)\right)$ and ${ }^{t}\left(\cos \left(\theta_{-}-\pi / 2\right), \sin \left(\theta_{-}-\right.\right.$ $\pi / 2))$.

(iv) For all $x \in \mathbb{R}$, it holds that $\varphi(x) \geq x \tan \left(\theta_{ \pm}-\pi / 2\right)$.

Proof. Lemma 4.1 implies that there exists a unique solution $\widetilde{\theta}$ of (4.4)-(4.6) up to translation. Let $\widetilde{\theta}$ be any solution. Then, $\widetilde{\theta}(x) \in\left(\theta_{-}, \theta_{+}\right), \widetilde{\theta}_{x}>0$. By (4.7), there exists $\alpha_{1}, \alpha_{2}>0$ such that

$$
\left|\widetilde{\theta}(x)-\theta_{ \pm}\right| \leq \alpha_{1} e^{-\alpha_{2}|x|} \quad \text { as } x \rightarrow \pm \infty .
$$

Then, by setting

$$
\widetilde{\varphi}(x)=\int_{0}^{x} \tan \left(\widetilde{\theta}(s)-\frac{\pi}{2}\right) d s
$$


one can easily check that $\widetilde{\varphi}$ satisfies (4.2)-(4.3). By (4.12), there exist two constants $C_{ \pm}$such that

$$
\lim _{x \rightarrow \pm \infty}\left[\widetilde{\varphi}(x)-x \tan \left(\theta_{ \pm}-\pi / 2\right)\right]=C_{ \pm} .
$$

Let ${ }^{t}\left(\rho_{1}, \rho_{2}\right)$ be the intersection between the two lines $y=x \tan \left(\theta_{ \pm}-\pi / 2\right)+C_{ \pm}$, then one can easily check that $\varphi(x)=\widetilde{\varphi}\left(x+\rho_{1}\right)-\rho_{2}$ is the unique solution of (4.2)-(4.3) satisfying

$$
\lim _{x \rightarrow \pm \infty}\left[\varphi(x)-x \tan \left(\theta_{ \pm}-\pi / 2\right)\right]=0 .
$$

In the sequel we will denote by $\theta$ the solution of (4.4)-(4.6) such that

$$
\varphi(x)=\varphi(0)+\int_{0}^{x} \tan (\theta(s)-\pi / 2) d s .
$$

Then (4.3) gives that

$$
\varphi^{\prime \prime}(x)=\frac{\theta^{\prime}(x)}{\cos ^{2}(\theta(x)-\pi / 2)}>0
$$

and since $\left(\varphi(x)-x \varphi^{\prime}(x)\right)^{\prime}=-x \varphi^{\prime \prime}(x)$, we have that

$$
\lim _{|x| \rightarrow+\infty}\left(\varphi(x)-x \varphi^{\prime}(x)\right)<\varphi(x)-x \varphi^{\prime}(x) \leq \varphi(0) .
$$

By (4.3) and (4.11), it holds that

$$
\lim _{|x| \rightarrow+\infty}\left(\varphi(x)-x \varphi^{\prime}(x)\right)=\lim _{|x| \rightarrow+\infty}\left(\varphi(x)-x \tan \left(\theta_{ \pm}-\pi / 2\right)\right)=0,
$$

which concludes the proof of (i). Note that (iv) directly follows from (i). Thus, (ii) follows from (4.3) and the fact that $\theta(x) \in\left(\theta_{-}, \theta_{+}\right)$. Also, the definition of ${ }^{t}\left(c_{1}, c_{2}\right)$ implies that

$$
\left(\begin{array}{c}
c_{1} \\
c_{2}
\end{array}\right) \cdot\left(\begin{array}{c}
\cos \theta_{ \pm} \\
\sin \theta_{ \pm}
\end{array}\right)=a \Psi\left(\theta_{ \pm}\right)>0
$$

which proves (iii).

In the rest of this section, we assume that $a>0$.

Next we consider the asymptotic stability of the traveling curved front. As in [13], we search for a supersolution and a subsolution of the following type:

$$
v(x, t)=\frac{1}{\alpha(t)} \varphi\left(\alpha(t)\left(x-c_{1} t\right)\right)+c_{2} t+\beta(t) .
$$

Putting

$$
z=\alpha(t)\left(x-c_{1} t\right)
$$


we have

$$
\begin{aligned}
L[v] & :=v_{t}-\frac{G_{1}\left(v_{x}\right) v_{x x}}{1+v_{x}^{2}}-G_{2}\left(v_{x}\right) \\
& =\frac{\alpha_{t}}{\alpha^{2}}\left(z \varphi^{\prime}(z)-\varphi(z)\right)-c_{1} \varphi^{\prime}(z)+c_{2}+\beta_{t}-\frac{\alpha G_{1}\left(\varphi^{\prime}(z)\right) \varphi^{\prime \prime}(z)}{1+\varphi^{\prime}(z)^{2}}-G_{2}\left(\varphi^{\prime}(z)\right) \\
& =\frac{\alpha_{t}}{\alpha^{2}}\left(z \varphi^{\prime}(z)-\varphi(z)\right)+\beta_{t}+(1-\alpha)\left\{c_{2}-c_{1} \varphi^{\prime}(z)-G_{2}\left(\varphi^{\prime}(z)\right)\right\} \\
& =\frac{\alpha_{t}}{\alpha^{2}}\left\{z \varphi^{\prime}-\varphi+\frac{\alpha^{2} \beta_{t}}{\alpha_{t}}+\frac{(1-\alpha) \alpha^{2}}{\alpha_{t}}\left(c_{2}-c_{1} \varphi^{\prime}-G_{2}\left(\varphi^{\prime}\right)\right)\right\}
\end{aligned}
$$

By this calculation we can construct the supersolution and subsolution.

Lemma 4.3. Let $v_{ \pm}(x, t)$ be defined by

$$
v_{ \pm}(x, t):=\frac{1}{\alpha_{ \pm}(t)} \varphi\left(\alpha_{ \pm}(t)\left(x-c_{1} t\right)\right)+c_{2} t+\beta_{ \pm}(t)
$$

where

$$
\begin{aligned}
& \alpha_{ \pm}(t):=1 \mp \delta e^{-\gamma t} \\
& \beta_{ \pm}(t):=\sigma\left(\frac{1}{\alpha_{ \pm}(0)}-\frac{1}{\alpha_{ \pm}(t)}\right) .
\end{aligned}
$$

Then, for any $\delta, \gamma$ are positive constants, there exists a non-negative constant $\sigma_{0}(\gamma, \delta)$ such that

$$
\lim _{\gamma \rightarrow 0} \sigma_{0}(\gamma, \delta)=0
$$

and $v_{+}$is a supersolution of (1.3) and $v_{-}$is a subsolution for $\sigma>\sigma_{0}(\gamma, \delta)$. Moreover, $v_{+}(x, t) \geq v_{-}(x, t)$ for any $x \in \mathbb{R}$ and $t \geq 0$.

Proof. By (4.15), we have

$$
L\left[v_{ \pm}\right]= \pm \frac{\delta \gamma e^{-\gamma t}}{\alpha_{ \pm}^{2}}\left\{z \varphi^{\prime}-\varphi+\sigma+\frac{\alpha_{ \pm}^{2}}{\gamma}\left(c_{2}-c_{1} \varphi^{\prime}-G_{2}\left(\varphi^{\prime}\right)\right)\right\} .
$$

Set

$$
\sigma_{0}(\gamma, \delta):=\sup _{z \in \mathbb{R}}\left\{-z \varphi^{\prime}(z)+\varphi(z)-\frac{(1-\delta)^{2}}{\gamma}\left(c_{2}-c_{1} \varphi^{\prime}(z)-G_{2}\left(\varphi^{\prime}(z)\right)\right)\right\} .
$$

Lemma 4.2 (i), (ii) and Lemma 4.1 immediately imply that $v_{+}$is a supersolution when $\sigma>\sigma_{0}(\gamma, \delta)$ and that $v_{-}$is a subsolution. Letting $z \rightarrow \pm \infty$, we see that $\sigma_{0}(\gamma, \delta) \geq 0$.

Next we shall show that for any $\varepsilon>0$ and $\delta \neq 1$, there exists a positive constant $\gamma_{0}$ such that

$$
\varphi(z)-z \varphi^{\prime}(z) \leq \varepsilon+\frac{(1-\delta)^{2}}{\gamma}\left(c_{2}-c_{1} \varphi^{\prime}(z)-G_{2}\left(\varphi^{\prime}(z)\right)\right)
$$


for $0<\gamma<\gamma_{0}$ and $z \in \mathbb{R}$, which in turn implies (4.17).

First, by (4.14), there exists a positive constant $R$ such that

$$
\varphi(z)-z \varphi^{\prime}(z) \leq \varepsilon \quad \text { for }|z| \geq R
$$

It follows from (4.2) and Lemma 4.2 (i) that, for $|z| \leq R$,

$$
c_{2}-c_{1} \varphi^{\prime}(z)-G_{2}\left(\varphi^{\prime}(z)\right)=\frac{G_{1}\left(\varphi^{\prime}(x)\right) \varphi^{\prime \prime}(x)}{1+\varphi^{\prime}(x)^{2}} \geq C_{R}>0
$$

for a suitable positive constant $C_{R}$. Then, Lemma 4.2 (i) implies that, for $\gamma$ close enough to 0 ,

$$
\begin{aligned}
\varphi(z)-z \varphi^{\prime}(z) & \leq \varphi(0) \leq \varepsilon+\frac{(1-\delta)^{2}}{\gamma} C_{R} \\
& \leq \varepsilon+\frac{(1-\delta)^{2}}{\gamma}\left(c_{2}-c_{1} \varphi^{\prime}(z)-G_{2}\left(\varphi^{\prime}(z)\right)\right)
\end{aligned}
$$

Next, we will show that $v_{+} \geq v_{-}$. The simple calculation leads us to

$$
v_{+}(x, 0)-v_{-}(x, 0)=\int_{\alpha_{+}(0)}^{\alpha_{-}(0)} \frac{1}{\alpha^{2}}\left(\varphi-z \varphi^{\prime}\right) d \alpha \geq 0 .
$$

Since $v_{+}$is a supersolution and $v_{-}$is a subsolution, we have proved that $v_{+}(x, t) \geq$ $v_{-}(x, t)$ for $t \geq 0$.

By the supersolutions and subsolutions in Lemma 4.3 we can show the local asymptotic stability of the traveling curved fronts by the similar argument to [13] which is based on $[5,6]$.

THEOREM 4.4. Assume that $u_{0}(x)$ satisfies

$$
\begin{aligned}
& \lim _{x \rightarrow \pm \infty}\left|u_{0}(x)-x \tan \left(\theta_{ \pm}-\pi / 2\right)\right|=0, \\
& u_{0}(x) \geq x \tan \left(\theta_{+}-\pi / 2\right) \quad \text { for } \quad x>0, \\
& u_{0}(x) \geq x \tan \left(\theta_{-}-\pi / 2\right) \quad \text { for } \quad x<0 .
\end{aligned}
$$

Then,

$$
\lim _{t \rightarrow \infty} \sup _{x \in \mathbb{R}}\left|u(x, t)-\varphi\left(x-c_{1} t\right)-c_{2} t\right|=0 .
$$

Note that, by Lemma 4.2 (iv), $v_{ \pm}(x, 0)$ also satisfy (4.19)-(4.20) and that the global existence of the solution $u(x, t)$ of (1.3) in time is guaranteed by the local existence of solutions of (1.3) and the existence of supersolutions and subsolutions in $[0, T]$ for all $T>0$. that

Proof. We shall show that, for any $\varepsilon>0$, there exists a large time $T$ such

$$
\varphi\left(x-c_{1} t\right)+c_{2} t-\varepsilon \leq u(x, t) \leq \varphi\left(x-c_{1} t\right)+c_{2} t+\varepsilon,
$$


for $t \geq T$ and $x \in \mathbb{R}$.

We first prove the upper estimate. By (4.18), there is a positive constant $R$ such that

$$
\left|u_{0}(x)-x \tan \left(\theta_{ \pm}-\pi / 2\right)\right| \leq \frac{\varepsilon}{3} \quad \text { for } \quad|x| \geq R
$$

Lemma 4.2 (iv) and (4.22) imply that, for $|x| \geq R$ and $\delta<1$,

$$
v_{+}(x, 0)+\frac{\varepsilon}{3}=\frac{1}{1-\delta} \varphi((1-\delta) x)+\frac{\varepsilon}{3} \geq x \tan \left(\theta_{ \pm}-\pi / 2\right)+\frac{\varepsilon}{3} \geq u_{0}(x) .
$$

Note that by Lemma $4.2(\mathrm{i}), \min _{x \in \mathbb{R}} \varphi(x)>0$ and choose $\delta$ close enough to 1 so that

$$
u_{0}(x) \leq \frac{1}{1-\delta} \min _{x \in \mathbb{R}} \varphi(x) \text { for }|x| \leq R .
$$

Then we have that for $|x| \leq R$

$$
\begin{aligned}
v_{+}(x, 0)+\frac{\varepsilon}{3} & =\frac{1}{1-\delta} \varphi((1-\delta) x)+\frac{\varepsilon}{3} \\
& \geq \frac{1}{1-\delta} \min _{x \in \mathbb{R}} \varphi(x)+\frac{\varepsilon}{3} \\
& \geq u_{0}(x) .
\end{aligned}
$$

Since by Lemma $4.3 v_{+}+\varepsilon / 3$ is also a supersolution, we have proved that for $\delta$ close enough to 1 and for all $\gamma>0$

$$
u(x, t) \leq v_{+}(x, t)+\frac{\varepsilon}{3} .
$$

Then take $\gamma$ so small that

$$
\frac{\delta \sigma_{0}(\gamma, \delta)}{(1-\delta)^{2}}<\frac{\varepsilon}{3}
$$

and choose $\sigma$ such that $\sigma_{0}(\gamma, \delta)<\sigma<\varepsilon(1-\delta)^{2} /(3 \delta)$. By setting $z=x-c_{1} t$ and by the definition of $v_{+}$, we have

$$
\begin{aligned}
v_{+}(x, t)-\varphi(z)-c_{2} t & =\frac{1}{\alpha_{+}(t)} \varphi\left(\alpha_{+}(t) z\right)-\varphi(z)+\sigma\left(\frac{1}{\alpha_{+}(0)}-\frac{1}{\alpha_{+}(t)}\right) \\
& \leq \int_{\alpha_{+}(t)}^{1} \frac{\varphi(\alpha z)-\alpha z \varphi^{\prime}(\alpha z)}{\alpha^{2}} d \alpha+\frac{\delta \sigma}{(1-\delta)^{2}} \\
& \leq \frac{\varphi(0)}{\alpha_{+}(t)^{2}}\left(1-\alpha_{+}(t)\right)+\frac{\delta \sigma}{(1-\delta)^{2}} \\
& \leq \frac{\varphi(0) \delta}{(1-\delta)^{2}} e^{-\gamma t}+\frac{\delta \sigma}{(1-\delta)^{2}}
\end{aligned}
$$

For $t \geq T_{1}:=\ln \left[(3 \varphi(0) \delta) /\left((1-\delta)^{2} \varepsilon\right)\right] / \gamma$ and for all $x \in \mathbb{R}$, we have

$$
u(x, t) \leq v_{+}(x, t)+\frac{\varepsilon}{3} \leq \varphi\left(x-c_{1} t\right)+c_{2} t+\varepsilon
$$


which proves the upper estimate. Note that inequality (4.23) is still establish even if $u_{0}$ does not satisfy (4.19)-(4.20).

Next we show the lower estimate. By Lemma 4.2, there is a positive constant $R$ such that

$$
\left|\varphi(x)-x \tan \left(\theta_{ \pm}-\pi / 2\right)\right| \leq \frac{\varepsilon}{3} \quad \text { for } \quad|x| \geq R .
$$

For all $\delta>0$ and all $|x| \geq R$, it holds that $|(1+\delta) x| \geq R$ and then (4.24) implies that

$$
v_{-}(x, 0)-\frac{\varepsilon}{3}=\frac{1}{1+\delta} \varphi((1+\delta) x)-\frac{\varepsilon}{3} \leq x \tan \left(\theta_{ \pm}-\pi / 2\right) \leq u_{0}(x),
$$

where the last inequality follows from (4.19)-(4.20). Moreover, for $|x| \leq R$, using Lemma 4.2 (i), it holds that

$$
\begin{aligned}
v_{-}(x, 0)-\frac{\varepsilon}{3} & =\frac{1}{1+\delta} \varphi((1+\delta) x)-\frac{\varepsilon}{3} \\
& \leq \frac{1}{1+\delta} \varphi(0)+x \varphi^{\prime}((1+\delta) x)-\frac{\varepsilon}{3} \\
& \leq \frac{1}{1+\delta} \varphi(0)+x \tan \left(\theta_{ \pm}-\pi / 2\right)-\frac{\varepsilon}{3} .
\end{aligned}
$$

Then let $\delta$ be so large that $\varphi(0) /(1+\delta) \leq \varepsilon / 3$, then (4.19)-(4.20) implies that for $|x| \leq R$,

$$
v_{-}(x, 0)-\frac{\varepsilon}{3} \leq u_{0}(x)
$$

Now, choose $\gamma$ and $\sigma$ satisfying

$$
\frac{\delta \sigma_{0}(\gamma, \delta)}{1+\delta}<\frac{\varepsilon}{3}, \quad \sigma_{0}(\gamma, \delta)<\sigma<\frac{(1+\delta) \varepsilon}{3 \delta}
$$

Since $v_{-}(x, t)-\varepsilon / 3$ is also a subsolution, Lemma 4.3 implies that, for $t \geq 0$,

$$
v_{-}(x, t)-\frac{\varepsilon}{3} \leq u(x, t) .
$$

Similarly as the upper estimate, we have

$$
\begin{aligned}
\varphi(z)+c_{2} t-v_{-}(x, t) & =\varphi(z)-\frac{1}{\alpha_{-}(t)} \varphi\left(\alpha_{-}(t) z\right)-\sigma\left(\frac{1}{\alpha_{-}(0)}-\frac{1}{\alpha_{-}(t)}\right) \\
& \leq \int_{1}^{\alpha_{-}(t)} \frac{\varphi(\alpha z)-\alpha z \varphi^{\prime}(\alpha z)}{\alpha^{2}} d \alpha+\frac{\delta \sigma}{1+\delta} \\
& \leq \varphi(0) \delta e^{-\gamma t}+\frac{\delta \sigma}{1+\delta} .
\end{aligned}
$$

If $T_{2}$ is large enough, it holds that $\varphi\left(x-c_{1} t\right)+c_{2} t-\varepsilon \leq u(x, t)$ for $t \geq T_{2}$, which proves inequality (4.21) for all $T \geq \max \left(T_{1}, T_{2}\right)$. 
Theorem 4.4 directly implies that the local asymptotic stability for the initial data satisfying (4.18)-(4.20). The assumption (4.18) is essential. It is shown in [13] that some solutions do not converge to the traveling wave, if (4.18) violates. The conditions (4.19) and (4.20) can be relaxed by introducing another subsolution as in $[13]$.

LEMMA 4.5. There exists $\varepsilon_{0}$ such that for any $\varepsilon \in\left(0, \varepsilon_{0}\right)$, there exist positive constants $\rho_{ \pm}$and two traveling curved fronts $y=\varphi_{ \pm}^{*}\left(x-c_{1} t\right)+c_{2} t$ satisfying

$$
\begin{aligned}
& \varphi_{+}^{*}(x) \text { is defined on }\left[-\rho_{+}, \infty\right), \text { and } \varphi_{-}^{*}(x) \text { is on }\left(-\infty, \rho_{-}\right] \text {respectively, } \\
& \lim _{x \rightarrow \pm \infty}\left(\varphi_{ \pm}^{*}(x)-x \tan \left(\theta_{ \pm}-\pi / 2\right)\right)=0 \\
& \lim _{x \rightarrow \mp \rho_{ \pm}} \frac{d}{d x} \varphi_{ \pm}^{*}(x)= \pm \infty \\
& \varphi_{+}^{*}(0)=\varphi_{-}^{*}(0)=-\varepsilon
\end{aligned}
$$

Furthermore, it holds that

$$
\left(\varphi_{ \pm}^{*}\right)^{\prime \prime}(z) \leq 0 \quad \text { and } \quad 0 \leq z\left(\varphi_{ \pm}^{*}\right)^{\prime}(z)-\varphi_{ \pm}^{*}(z) \leq \varepsilon .
$$

Proof. Using Lemma 4.1, one shows that there exist solutions $\theta_{ \pm}^{*}$ of (4.4) such that

$$
\begin{array}{ll}
\left(\theta_{-}^{*}\right)^{\prime}=f\left(\theta_{-}^{*}\right), & \theta_{-}^{*}\left(\rho_{-}\right)=0, \quad \theta_{-}^{*}(-\infty)=\theta_{-}, \\
\left(\theta_{+}^{*}\right)^{\prime}=f\left(\theta_{+}^{*}\right), \quad \theta_{+}^{*}\left(-\rho_{+}\right)=\pi, \quad \theta_{+}^{*}(\infty)=\theta_{+} .
\end{array}
$$

By an appropriate shifts in $x$ and $y$, we can easily check the existence of $\varphi_{ \pm}^{*}$. Moreover, by denoting by $\theta_{ \pm}^{*}$ the orbits such that

$$
\left(\varphi_{ \pm}^{*}\right)^{\prime}=\tan \left(\theta_{ \pm}^{*}-\pi / 2\right)
$$

it holds that

$$
\left(\varphi_{ \pm}^{*}\right)^{\prime \prime}=\frac{\left(\theta_{ \pm}^{*}\right)^{\prime}}{\cos ^{2}\left(\theta_{ \pm}^{*}-\pi / 2\right)}<0,
$$

and the equality $\left(\varphi_{ \pm}^{*}(z)-z\left(\varphi_{ \pm}^{*}\right)^{\prime}(z)\right)^{\prime}=-z\left(\varphi_{ \pm}^{*}\right)^{\prime \prime}$ implies that

$$
\varphi_{ \pm}^{*}(z)-z\left(\varphi_{ \pm}^{*}\right)^{\prime}(z) \geq-\varepsilon,
$$

which concludes the proof.

Note that $\tan \left(\theta_{-}^{*}-\pi / 2\right) \leq \tan \left(\theta_{-}-\pi / 2\right)$ and that $\tan \left(\theta_{+}^{*}-\pi / 2\right) \geq \tan \left(\theta_{+}-\right.$ $\pi / 2$ ) so that

$$
\begin{aligned}
& \varphi_{+}^{*}(z)=\varphi_{+}^{*}(0)+\int_{0}^{z} \tan \left(\theta_{+}^{*}-\pi / 2\right) d s \geq-\varepsilon+z \tan \left(\theta_{+}-\pi / 2\right) \text { for } z \geq 0, \\
& \varphi_{-}^{*}(z)=\varphi_{-}^{*}(0)+\int_{0}^{z} \tan \left(\theta_{-}^{*}-\pi / 2\right) d s \geq-\varepsilon+z \tan \left(\theta_{-}-\pi / 2\right) \text { for } z \leq 0 .
\end{aligned}
$$


Using $\varphi_{ \pm}^{*}$ we can construct the following subsolution.

Lemma 4.6. Let $v^{-}$be defined by

$$
v^{-}(x, t):= \begin{cases}\frac{1}{\alpha^{*}(t)} \varphi_{+}^{*}\left(\alpha^{*}(t)\left(x-c_{1} t\right)\right)+c_{2} t+\beta^{*}(t), & \text { for } x-c_{1} t \geq 0 \\ \frac{1}{\alpha^{*}(t)} \varphi_{-}^{*}\left(\alpha^{*}(t)\left(x-c_{1} t\right)\right)+c_{2} t+\beta^{*}(t), & \text { for } x-c_{1} t \leq 0\end{cases}
$$

where

$$
\begin{aligned}
& \alpha^{*}(t):=1-\delta^{*} e^{-\gamma^{*} t}, \\
& \beta^{*}(t):=-\sigma^{*}\left(\frac{1}{\alpha^{*}(0)}-\frac{1}{\alpha^{*}(t)}\right) .
\end{aligned}
$$

For any positive constants $\delta^{*}, \gamma^{*}$, there exists a positive constant $\sigma_{0}^{*}(\gamma, \delta)$ such that $v^{-}$is a subsolution of (1.3) for $\sigma^{*}>\sigma_{0}^{*}\left(\gamma^{*}, \delta^{*}\right)$. Moreover,

$$
\lim _{\gamma^{*} \rightarrow 0} \sigma_{0}^{*}\left(\gamma^{*}, \delta^{*}\right)=0
$$

Proof. We consider the case on $\left[-\rho_{+}, \infty\right)$. It follows from $(4.15)$ that

$$
L\left[v^{-}\right]=-\frac{\delta^{*} \gamma^{*} e^{-\gamma t}}{\left(\alpha^{*}\right)^{2}}\left\{-z\left(\varphi_{+}^{*}\right)^{\prime}+\varphi_{+}^{*}+\sigma^{*}+\frac{\left(\alpha^{*}\right)^{2}}{\gamma^{*}}\left(-c_{2}+c_{1}\left(\varphi_{+}^{*}\right)^{\prime}+G_{2}\left(\left(\varphi_{+}^{*}\right)^{\prime}\right)\right)\right\},
$$

so that $L\left[v^{-}\right] \leq 0$ for all $\sigma^{*} \geq \sigma_{0}^{*}\left(\gamma^{*}, \delta^{*}\right)$ where

$$
\sigma_{0}^{*}\left(\gamma^{*}, \delta^{*}\right):=\sup _{z \in \mathbb{R}}\left\{z\left(\varphi_{+}^{*}\right)^{\prime}-\varphi_{+}^{*}-\frac{\left(1-\delta^{*}\right)^{2}}{\gamma^{*}}\left(-c_{2}+c_{1}\left(\varphi_{+}^{*}\right)^{\prime}+G_{2}\left(\left(\varphi_{+}^{*}\right)^{\prime}\right)\right)\right\} .
$$

By letting $z \rightarrow+\infty$, we see that $\sigma_{0}^{*}\left(\gamma^{*}, \delta^{*}\right) \geq 0$. To show (4.27), we need to prove that, for any $\varepsilon^{\prime}>0$, there exists $\gamma_{0}^{*}$ such that

$$
z\left(\varphi_{+}^{*}\right)^{\prime}-\varphi_{+}^{*} \leq \varepsilon^{\prime}+\frac{\left(1-\delta^{*}\right)^{2}}{\gamma^{*}}\left(-c_{2}+c_{1}\left(\varphi_{+}^{*}\right)^{\prime}+G_{2}\left(\left(\varphi_{+}^{*}\right)^{\prime}\right)\right)
$$

for $0<\gamma^{*}<\gamma_{0}^{*}$ and $z \in \mathbb{R}$. By the similar argument in the proof of Lemma 4.3, we see the above inequality.

We can perform a similar computation for $\varphi_{-}^{*}$. Then, the lemma follows from the fact that the maximum of subsolutions is also a subsolution.

We give a simpler proof of the global stability than in [13].

THEOREM 4.7. Let $u_{0}(x)$ satisfy (4.18) and $u(x, t)$ be a solution of (4.1) with $u(x, 0)=u_{0}(x)$. Then,

$$
\lim _{t \rightarrow \infty} \sup _{x \in \mathbb{R}}\left|u(x, t)-\varphi\left(x-c_{1} t\right)-c_{2} t\right|=0 .
$$


Proof. In view of Theorem 4.4, we only need to show that, for any $\varepsilon>0$, there exists a time $T$ such that

$$
x \tan \left(\theta_{ \pm}-\pi / 2\right) \leq u\left(x+c_{1} T, T\right)-c_{2} T+3 \varepsilon \quad \text { for } \quad x \in \mathbb{R} .
$$

To that purpose, we use a similar argument as in Theorem 4.4. Let $\varepsilon>0$ be small enough and take $\varphi_{ \pm}^{*}$ as in Lemma 4.5. First, we can choose $R>0$ such that

$$
x \tan \left(\theta_{ \pm}-\pi / 2\right)-\varepsilon<u_{0}(x) \text { for }|x| \geq R .
$$

Then, choose $\eta>0$ so that $\varphi_{ \pm}^{*}( \pm x) \leq-\varepsilon / 2$ for all $0 \leq x \leq \eta$. Define

$$
M_{R}=\sup _{x \in[-R, R]}\left|u_{0}(x)\right|
$$

and choose $\delta^{*} \in(0,1)$ close enough to 1 so that

$$
\left(1-\delta^{*}\right) R<\eta, \quad \text { and } \quad \frac{\varepsilon}{2\left(1-\delta^{*}\right)} \geq M_{R}
$$

Then, if $|x| \leq R$, it holds that $\left|\left(1-\delta^{*}\right) x\right| \leq \eta$ and then by Lemma 4.6

$$
v^{-}(x, 0)=\frac{1}{1-\delta^{*}} \varphi_{ \pm}^{*}\left(\left(1-\delta^{*}\right) x\right) \leq \frac{1}{1-\delta^{*}} \times \frac{-\varepsilon}{2} \leq-M_{R} \leq u_{0}(x),
$$

which together with (4.29) and (4.25) implies that

$$
v^{-}(x, 0)-\varepsilon \leq u_{0}(x) \text { for all } x \in \mathbb{R} .
$$

Then for all positive constants $\gamma^{*}$ and $\sigma^{*}$ such that $\sigma^{*} \geq \sigma_{0}^{*}\left(\gamma^{*}, \delta^{*}\right)$, Lemma 4.6 implies that

$$
v^{-}(x, t)-\varepsilon \leq u(x, t)
$$

for any $x \in R$ and $t \geq 0$.

By (4.27), we can choose $\gamma^{*}$ and $\sigma^{*}$ small enough to satisfy

$$
\frac{2 \sigma_{0}^{*}\left(\gamma^{*}, \delta^{*}\right) \delta^{*}}{1-\delta^{*}} \leq \frac{2 \sigma^{*} \delta^{*}}{1-\delta^{*}}<\varepsilon .
$$

Using (4.26), we have, for $x>0$,

$$
\begin{aligned}
v^{-}\left(x+c_{1} t, t\right)-c_{2} t & =\frac{1}{\alpha^{*}(t)} \varphi_{+}^{*}\left(\alpha^{*}(t) x\right)+\beta^{*}(t) \\
& \geq \frac{1}{\alpha^{*}(t)}\left(-\varepsilon+\alpha^{*}(t) x \tan \left(\theta_{+}-\pi / 2\right)\right)-\frac{\sigma^{*} \delta^{*}}{1-\delta^{*}} \\
& \geq x \tan \left(\theta_{+}-\pi / 2\right)-\frac{\varepsilon}{\alpha^{*}(t)}-\frac{\sigma^{*} \delta^{*}}{1-\delta^{*}} .
\end{aligned}
$$


Then, for $t \geq\left(\ln 3 \delta^{*}\right) / \gamma^{*}$, it holds that

$$
v^{-}\left(x+c_{1} t, t\right)-c_{2} t \geq x \tan \left(\theta_{+}-\pi / 2\right)-2 \varepsilon .
$$

Similarly it holds that, for $x<0$ and $t$ large enough,

$$
v^{-}\left(x+c_{1} t, t\right)-c_{2} t \geq x \tan \left(\theta_{-}-\pi / 2\right)-2 \varepsilon .
$$

Combining (4.30)-(4.32) immediately implies (4.28).

\section{Level Set Methods}

In this section, we give an application of our result to movement by anisotropic curvature in the plane. More precisely, let $\Gamma_{0}$ be a curve in $\mathbb{R}^{2}$ satisfying:

$\Gamma_{0}$ possesses asymptotic lines $y=x \tan \left(\theta_{ \pm}-\pi / 2\right)$ as $x \rightarrow \pm \infty$.

Namely, we suppose that there are two functions $\zeta_{1}, \zeta_{2} \in C(\mathbb{R})$ such that $\zeta_{1}, \zeta_{2}$ satisfy hypothesis (H3) and

$$
\begin{gathered}
\Gamma_{0} \subset\left\{(x, y) \in \mathbb{R}^{2} \mid \zeta_{1}(x) \leq y \leq \zeta_{2}(x)\right\}, \\
\lim _{x \rightarrow \pm \infty}\left(\zeta_{1}(x)-x \tan \left(\theta_{ \pm}-\pi / 2\right)\right)=0, \\
\lim _{x \rightarrow \pm \infty}\left(\zeta_{2}(x)-x \tan \left(\theta_{ \pm}-\pi / 2\right)\right)=0 .
\end{gathered}
$$

Let $U_{0}$ be a continuous function such that

$$
\begin{aligned}
& \left\{(x, y) \in \mathbb{R}^{2} \mid U_{0}(x, y)=0\right\}=\Gamma_{0}, \\
& U_{0}(x, y)=1 \quad \text { if } y \geq \zeta_{2}(x)+1, \quad U_{0}(x, y)=-1 \quad \text { if } y \leq \zeta_{1}(x)-1
\end{aligned}
$$

and such that there exist $\nu \in[0,(1+\sqrt{5}) / 2)$ and a modulus of continuity $m$ such that

$$
\left|U_{0}(x, y)-U_{0}\left(x^{\prime}, y^{\prime}\right)\right| \leq m\left(\left(1+|x|+\left|x^{\prime}\right|+|y|+|y|^{\prime}\right)^{\nu}\right)\left(\left|x-x^{\prime}\right|+\left|y-y^{\prime}\right|\right)
$$

for all $x, y \in \mathbb{R}$.

Then, we prove the following result.

THEOREM 5.1. Let $\Gamma_{0}$ be as above and $U$ be the unique solution of (1.2) with $U(x, y, 0)=U_{0}(x, y)$. Set

$$
\Gamma_{t}:=\left\{(x, y) \in \mathbb{R}^{2} \mid U(x, y, t)=0\right\} .
$$

Then, for any $\varepsilon>0$, there exists $T>0$ such that for all $t \geq T$

$$
\Gamma_{t} \subset\left\{(x, y) \in \mathbb{R}^{2}|| y-\varphi\left(x-c_{1} t\right)-c_{2} t \mid \leq \varepsilon\right\}
$$


Note that the existence of a solution as well as a comparison principle is already known (see $[2,10]$ for instance).

Proof. Let $u^{-}$be the solution of (4.1) with $u^{-}(x, 0)=\zeta_{1}(x)$. Since $u_{0}^{-}$satisfies (4.18), by Theorem 4.7, it holds that

$$
\lim _{t \rightarrow \infty} \sup _{x \in \mathbb{R}}\left|u^{-}(x, t)-\varphi\left(x-c_{1} t\right)-c_{2} t\right|=0 .
$$

Moreover, setting $U_{0}^{+}(x, y)=\sup \left(y-\zeta_{1}(x), U_{0}(x, y)\right)$, we have that $U_{0}^{+}(x, y) \geq$ $U_{0}(x, y)$ for all $(x, y) \in \mathbb{R}^{2}$. If $U^{+}$denotes the solution of $(1.2)$ with $U^{+}(x, y, 0)=$ $U_{0}^{+}(x, y)$, the comparison principle for (1.2) implies that $U^{+}(x, y, t) \geq U(x, y, t)$ for all $t \geq 0$.

Since $U_{0}^{+}(x, y)=0$ if and only if $y=\zeta_{1}(x)$, it holds that

$$
\Gamma_{t}^{+}:=\left\{(x, y) \in \mathbb{R}^{2} \mid U^{+}(x, y, t)=0\right\}=\left\{(x, y) \in \mathbb{R}^{2} \mid y=u^{-}(x, t)\right\},
$$

so that

$$
U\left(x, u^{-}(x, t), t\right) \leq 0 .
$$

Similarly, one can construct a solution $u^{+}$of (4.1) such that

$$
\lim _{t \rightarrow \infty} \sup _{x \in \mathbb{R}}\left|u^{+}(x, t)-\varphi\left(x-c_{1} t\right)-c_{2} t\right|=0,
$$

and such that

$$
U\left(x, u^{+}(x, t), t\right) \geq 0 .
$$

Then, for all $t \geq 0$, the equations (5.1) and (5.2) imply that if $(x, y) \in \Gamma_{t}$ then $u^{-}(x, t) \leq y \leq u^{+}(x, t)$ which by letting $t \rightarrow \infty$ concludes the proof.

\section{Singular Limit of Traveling Curved Fronts and Crystalline Motions}

In this section we consider the profile of the traveling waves when $\Psi$ includes the small parameter $\varepsilon>0$.

We assume that $\Psi=\Psi(\theta, \varepsilon)$ is a $\pi$-periodic function in $\theta$ which belongs to $C^{2}\left(\mathbb{R} \times\left(0, \varepsilon_{0}\right], \mathbb{R}\right) \cap C^{0}\left(\mathbb{R} \times\left[0, \varepsilon_{0}\right], \mathbb{R}\right)$ with some positive constant $\varepsilon_{0}$ and satisfies (H2) where $\lambda_{1}$ and $\lambda_{2}$ are independent of $\varepsilon$ and $\lambda_{3}$ and $\lambda_{4}$ depends on $\varepsilon$. We also write $f(\theta, \varepsilon)$ instead of $f(\theta)$ to emphasize the dependence on $\varepsilon$. Assume furthermore that

(H4) There exist $0 \leq \theta_{1}<\theta_{2}<\cdots<\theta_{m}<2 \pi$ and positive constants $m_{j}$ such that

$$
\begin{aligned}
& \Psi(\theta, \varepsilon)+\Psi_{\theta \theta}(\theta, \varepsilon) \rightarrow \sum_{j=1}^{m} m_{j} \delta\left(\theta-\theta_{j}\right) \quad \text { in the distribution sense as } \varepsilon \downarrow 0, \\
& \lim _{\varepsilon \downarrow 0}\left[\Psi(\theta, \varepsilon)+\Psi_{\theta \theta}(\theta, \varepsilon)\right]=0 \quad \text { if } \theta \notin\left\{\theta_{j}\right\}_{j=1}^{m},
\end{aligned}
$$


(H5) There are positive integers $j_{1}$ and $j_{2}$ such that $1 \leq j_{1} \leq j_{2} \leq m$ and $\theta_{-}<\theta_{j_{1}} \leq \theta_{j_{2}}<\theta_{+}$and $\theta_{j_{1}-1}<\theta_{-}$, if $j_{1} \geq 2$, and $\theta_{+}<\theta_{j_{2}+1}$, if $j_{2} \leq m-1$.

Since the velocity $\left(c_{1}, c_{2}\right)$ given by $(4.8)$ also depends continuously on $\varepsilon$, we will also write $\left(c_{1}^{\varepsilon}, c_{2}^{\varepsilon}\right)$. We also write $C_{ \pm}, \rho_{1}$ and $\rho_{2}$ as in the proof of Lemma 4.2 with $f=f(\theta, \varepsilon)$ by $C_{ \pm}^{\varepsilon}, \rho_{1}^{\varepsilon}$ and $\rho_{2}^{\varepsilon}$ respectively.

In [9, Corollary 1.3], it was proved that the motion by crystalline energy (6.4) below is a limit of the regularized problem (1.3). More precisely, the evolving solution of (1.3) with the periodic boundary conditions converges to the admissible solution of (6.4) locally uniformly as $\varepsilon \downarrow 0$ provided that the initial function $u_{0}^{\varepsilon}$ converges to $u_{0}^{0}$ uniformly. For the traveling wave solutions of (1.3), we can show that they converge to those of the corresponding crystalline motion in the following sense.

Theorem 6.1. Assume (H1)-(H5). Let $\varphi(x, \varepsilon)$ be a traveling curved front given in Lemma 4.2 and $L_{j}\left(j=1, \ldots, j_{2}-j_{1}+1\right)$ a positive constant given by

$$
L_{j}:=\frac{m_{j+j_{1}-1} \Psi\left(\theta_{j+j_{1}-1}, 0\right)}{c_{1}^{0} \cos \theta_{j+j_{1}-1}+c_{2}^{0} \sin \theta_{j+j_{1}-1}-a \Psi\left(\theta_{j+j_{1}-1}, 0\right)} .
$$

Then there exist constants $x_{1}, \ldots, x_{j_{2}-j_{1}+1}$ with

$$
x_{j+1}-x_{j}=L_{j} \sin \theta_{j+j_{1}-1}
$$

such that $\varphi(x, \varepsilon)$ converges to $\widehat{\varphi}(x)$ as $\varepsilon \downarrow 0$ where

$$
\widehat{\varphi}^{\prime}(x)=\left\{\begin{array}{ll}
\tan \left(\theta_{-}-\pi / 2\right) & \left(-\infty, x_{1}\right], \\
\tan \left(\theta_{j+j_{1}-1}-\pi / 2\right) & \left(x_{j}, x_{j+1}\right], \\
\tan \left(\theta_{+}-\pi / 2\right) & \left(x_{j_{2}-j_{1}+1}, \infty\right)
\end{array} \quad\left(j=1, \ldots, j_{2}-j_{1} \quad \text { if } j_{1}<j_{2}\right)\right.
$$

Moreover, if $j_{1}<j_{2}$, then the normal velocity $V_{j}$ of the facet between $\left(x_{j}, \widehat{\varphi}\left(x_{j}\right)\right)$ and $\left(x_{j+1}, \widehat{\varphi}\left(x_{j+1}\right)\right)\left(j=1, \ldots, j_{2}-j_{1}\right)$ satisfies the following crystalline motion

$$
V_{j}=\left(\frac{m_{j+j_{1}-1}}{L_{j}}+a\right) \Psi\left(\theta_{j+j_{1}-1}, 0\right) .
$$

Proof. For simplicity, we set

$$
d_{j}:=L_{j} \sin \theta_{j+j_{1}-1}
$$

First consider the case where $j_{1}<j_{2}$. It follows from (4.4) and the definition of $f$ that

$$
x=x(\widetilde{\theta}, \varepsilon):=x_{1}+\int_{\left(\theta_{j_{1}}+\theta_{-}\right) / 2}^{\widetilde{\theta}} \frac{d s}{f(s, \varepsilon)}
$$


where $x_{1}$ is specified later. By $(\mathrm{H} 4)$, we have

$$
\begin{aligned}
\int_{\theta_{j+j_{1}-1}-\delta}^{\theta_{j+j_{1}-1}+\delta} \frac{d s}{f(s, \varepsilon)} & =\int_{\theta_{j+j_{1}-1}-\delta}^{\theta_{j+j_{1}-1}+\delta} \frac{\Psi(s, \varepsilon)\left(\Psi(s, \varepsilon)+\Psi^{\prime \prime}(s, \varepsilon)\right) \sin s}{c_{1}^{\varepsilon} \cos s+c_{2}^{\varepsilon} \sin s-a \Psi(s, \varepsilon)} d s \\
& \longrightarrow \frac{\Psi\left(\theta_{j+j_{1}-1}, 0\right) m_{j} \sin \theta_{j+j_{1}-1}}{c_{1}^{0} \cos \theta_{j+j_{1}-1}+c_{2}^{0} \sin \theta_{j+j_{1}-1}-a \Psi\left(\theta_{j+j_{1}-1}, 0\right)}=d_{j}
\end{aligned}
$$

as $\varepsilon$ tends to 0 for sufficiently small positive $\delta$. It turns out that

$$
\lim _{\varepsilon \downarrow 0} x(\widetilde{\theta}, \varepsilon)= \begin{cases}x_{1} & \left(\theta_{-}<\tilde{\theta}<\theta_{j_{1}}\right), \\ x_{j} & \left(\theta_{j+j_{1}-2}<\widetilde{\theta}<\theta_{j+j_{1}-1}\right), \quad\left(j=2, \ldots, j_{2}-j_{1}+1\right) \\ x_{j_{2}-j_{1}+1} & \left(\theta_{j_{2}}<\widetilde{\theta}<\theta_{+}\right),\end{cases}
$$

where $x_{j+1}:=x_{j}+d_{j}\left(j=1, \ldots, j_{2}-j_{1}+1\right)$. Thus, the inverse function $\tilde{\theta}(x, \varepsilon)$ of $x(\widetilde{\theta}, \varepsilon)$ converges to

$$
\lim _{\varepsilon \downarrow 0} \widetilde{\theta}(x, \varepsilon)= \begin{cases}\theta_{-} & \left(x<x_{1}\right), \\ \theta_{j+j_{1}-1} & \left(x_{j}<x<x_{j+1}\right), \quad\left(j=1, \ldots, j_{2}-j_{1}\right) \\ \theta_{+} & \left(x_{j_{2}-j_{1}+2}<x\right) .\end{cases}
$$

Recall that $\widetilde{\varphi}(x, \varepsilon)$ can be defined by $\widetilde{\theta}$. By $(4.10),(4.9)$ and $(\mathrm{H} 5)$, we have

$$
\liminf _{\varepsilon \downarrow 0} \frac{f^{\prime}\left(\theta_{-}\right)}{a}>0, \quad \limsup _{\varepsilon \downarrow 0} \frac{f^{\prime}\left(\theta_{+}\right)}{a}<0 .
$$

These inequalities and the fact that

$$
C_{ \pm}^{\varepsilon}=\lim _{x \rightarrow \pm \infty} \int_{0}^{x}\left(\tan (\widetilde{\theta}(s, \varepsilon)-\pi / 2)-\tan \left(\theta_{ \pm}-\pi / 2\right)\right) d s .
$$

imply that $C_{ \pm}^{\varepsilon}$ converges to $C_{ \pm}^{0}$ as $\varepsilon \downarrow 0$. Since $\rho_{i}^{\varepsilon}$ continuously depends only on $C_{ \pm}^{\varepsilon}$ and $\theta_{ \pm}, \rho_{i}^{\varepsilon}$ also converges to $\rho_{i}^{0}$ as $\varepsilon \downarrow 0$. Thus the traveling wave $\varphi(x, \varepsilon)$ converges to the segment with the slope $\tan \left(\theta_{j+j_{1}-1}-\pi / 2\right)$ in the interval $\left[x_{j}, x_{j+1}\right]$ $\left(j=1, \ldots, j_{2}-j_{1}+1\right)$ and $x_{1}$ is uniquely defined by $\rho_{j}^{0}(j=1,2)$. Each facet between $\left(x_{j}, \widehat{\varphi}\left(x_{j}\right)\right)$ and $\left(x_{j+1}, \widehat{\varphi}\left(x_{j+1}\right)\right)\left(j=1, \ldots, j_{2}-j_{1}+1\right)$ moves with constant velocity. The length of each facet is $L_{j}=d_{j} / \sin \theta_{j+j_{1}-1}$ and its normal vector is

$$
n_{j}:=\left(\begin{array}{c}
\cos \theta_{j+j_{1}-1} \\
\sin \theta_{j+j_{1}-1}
\end{array}\right) \text {. }
$$

The normal velocity is

$$
V_{j}:=n_{j} \cdot\left(\begin{array}{c}
c_{1}^{0} \\
c_{2}^{0}
\end{array}\right)
$$

By (6.1), we have

$$
\begin{aligned}
V_{j} & =c_{1}^{0} \cos \theta_{j+j_{1}-1}+c_{2}^{0} \sin \theta_{j+j_{1}-1} \\
& =\left(\frac{m_{j+j_{1}-1}}{L_{j}}+a\right) \Psi\left(\theta_{j+j_{1}-1}, 0\right) .
\end{aligned}
$$


This shows that the traveling front of (1.1) converges to the traveling faceting (6.3) governed by the crystalline motion (6.4) (see [1, Section 10.3] or [11, Section 12.5]).

In the case where $j_{1}=j_{2}$, the similar argument implies the existence of $x_{1}$ and $x_{2}$ satisfying (6.2). This completes the proof.

In this situation, every juncture point moves with the constant velocity $\left(c_{1}^{0}, c_{2}^{0}\right)$. We remark that if there are no $\theta_{j}$ between $\left(\theta_{-}, \theta_{+}\right)$, the traveling wave converges to the faceting curve with two facets $\max \left\{x \tan \left(\theta_{-}-\pi / 2\right), x \tan \left(\theta_{+}-\pi / 2\right)\right\}$. We also note that the facets on $\left(-\infty, x_{1}\right]$ and $\left[x_{j_{2}}, \infty\right)$ are not admissible, because $\theta_{ \pm}$ do not belong to $\left\{\theta_{j}\right\}$ by (H5). It is different from the situation on the periodic boundary condition obtained in [9].

We consider the following example. Set

$$
\Psi(\theta, \varepsilon):=\sqrt{\cos ^{2}(\theta-\pi / 4)+\varepsilon}+\sqrt{\sin ^{2}(\theta-\pi / 4)+\varepsilon} .
$$

Then,

$$
\Psi(\theta, \varepsilon)+\Psi_{\theta \theta}(\theta, \varepsilon)=\frac{\varepsilon(1+\varepsilon)}{\left(\cos ^{2}(\theta-\pi / 4)+\varepsilon\right)^{3 / 2}}+\frac{\varepsilon(1+\varepsilon)}{\left(\sin ^{2}(\theta-\pi / 4)+\varepsilon\right)^{3 / 2}} .
$$

Setting

$$
\theta_{j}:=\frac{\pi+2(j-1) \pi}{4}, \quad(j=1,2,3,4)
$$

we get

$$
\Psi\left(\theta_{j}, \varepsilon\right)+\Psi_{\theta \theta}\left(\theta_{j}, \varepsilon\right)=\frac{\varepsilon(1+\varepsilon)}{(1+\varepsilon)^{3 / 2}}+\frac{\varepsilon(1+\varepsilon)}{\varepsilon^{3 / 2}} \rightarrow \infty \quad \text { as } \varepsilon \rightarrow 0 .
$$

Using the change of variables $\sin s=\sqrt{\varepsilon} \tan \eta$, we can check that

$$
\int_{-\delta}^{\delta} \frac{\varepsilon(1+\varepsilon)}{\left(\sin ^{2} s+\varepsilon\right)^{3 / 2}} d s=\int_{-\arctan (\sin \delta / \sqrt{\varepsilon})}^{\arctan (\sin \delta / \sqrt{\varepsilon})} \frac{(1+\varepsilon) \cos \eta}{\sqrt{1-\varepsilon \tan ^{2} \eta}} d \eta \rightarrow 2 \quad \text { as } \quad \varepsilon \rightarrow 0
$$

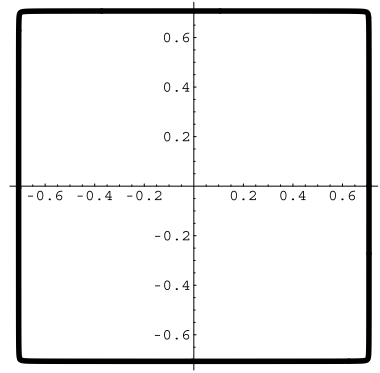

(a)

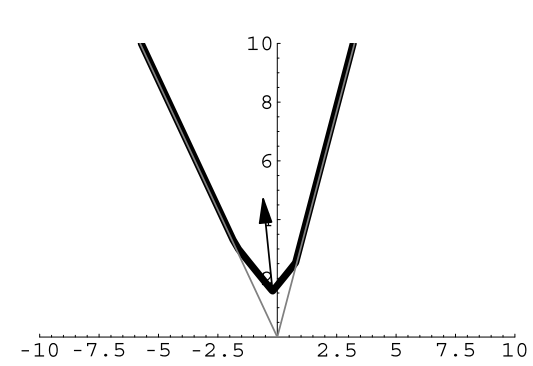

(b)

Fig. 1. The traveling curved front with (6.6). (a) The frank diagram $|\xi|=1 / \Psi(\theta)$; (b) the corresponding traveling curved front. The arrow indicates its velocity. 
for small $\delta>0$. This implies that (H4) and (H5) hold and that $m_{j}=2$. The traveling faceting curve with $\theta_{-}=\pi / 6$ and $\theta_{+}=9 \pi / 10$ is in Fig. 1.

Acknowledgement. The second author was partially supported by Grant-inAid for Encouragement of Young Scientists No. 15740076 (Japan).

\section{References}

[1] S. Angenent and M.E. Gurtin, Multiphase thermomechanics with interfacial structure. II. Evolution of an isothermal interface. Arch. Rational Mech. Anal., 108 (1989), 323-391.

[ 2 ] G. Barles, S. Biton, M. Bourgoing and O. Ley, Uniqueness results for quasilinear parabolic equations through viscosity solutions' methods. Calc. Var. Partial Differential Equations, 18 (2003), 159-179.

[ 3 ] G. Bellettini and M. Paolini, Anisotropic motion by mean curvature in the context of Finsler geometry. Hokkaido Math. J., 25 (1996), 537-566.

[ 4 ] M. Beneš, D. Hilhorst and R. Weidenfeld, Interface dynamics for an anisotropic Allen-Cahn equation. Proceedings of the Conference on Nonlinear Elliptic and Parabolic Problems, Banach Center Publications 66, 2004, 39-45.

[ 5 ] X. Chen, Generation and propagation of interfaces for reaction-diffusion equations. J. Differential Equations, 96 (1992), 116-141.

[6] X. Chen, Existence, uniqueness, and asymptotic stability of traveling waves in nonlocal evolution equations. Advances in Differential Equations, 2 (1997), 125-160.

[ 7 ] M.G. Crandall, H. Ishii and P.-L. Lions, User's guide to viscosity solutions of second order partial differential equations. Bull. Amer. Math. Soc. (N.S.), 27 (1992), 1-67.

[ 8 ] K. Deckelnick, C.M. Elliott and G. Richardson, Long time asymptotics for forced curvature flow with applications to the motion of a superconducting vortex. Nonlinearity, 10 (1997), 655-678.

[ 9 ] M.-H. Giga and Y. Giga, Stability for evolving graphs by nonlocal weighted curvature. Comm. in Partial Differential Equations, 24 (1999), 109-184.

[10] Y. Giga, Surface Evolution Equations. To appear.

[11] M.E. Gurtin, Thermomechanics of Evolving Phase Boundaries in the Plane. Oxford Science Publ., 1993.

[12] H. Ninomiya and M. Taniguchi, Traveling curved fronts of a mean curvature flow with constant driving force. Free Boundary Problems: Theory and Applications, I, GAKUTO Internat. Ser. Math. Sci. Appl. 13, 2000, 206-221.

[13] H. Ninomiya and M. Taniguchi, Stability of traveling curved fronts in a curvature flow with driving force. Methods and Application of Analysis, 8 (2001), 429-450.

[14] J.E. Taylor and J.W. Cahn, Diffuse interfaces with sharp corners and facets: Phase field models with strongly anisotropic surfaces. Physica D, 112 (1998), 381-411. 